\title{
Ligand-independent requirements of steroid receptors EcR and USP for cell survival
}

\author{
A Mansilla ${ }^{1,3}$, FA Martín ${ }^{1,3}$, D Martín $^{2}$ and A Ferrús ${ }^{1}$
}

The active form of the Drosophila steroid hormone ecdysone, 20-hydroxyecdysone (20E), binds the heterodimer EcR/USP nuclear receptor to regulate target genes that elicit proliferation, cell death and differentiation during insect development. Although the $20 \mathrm{E}$ effects are relatively well known, the physiological relevance of its receptors remains poorly understood. We show here that the prothoracic gland (PG), the major steroid-producing organ of insect larvae, requires EcR and USP to survive in a critical period previous to metamorphosis, and that this requirement is $20 \mathrm{E}$-independent. The cell death induced by the downregulation of these receptors involves the activation of the JNK-encoding basket gene and it can be rescued by upregulating EcR isoforms which are unable to respond to 20E. Also, while PG cell death prevents ecdysone production, blocking hormone synthesis or secretion in normal PG does not lead to cell death, demonstrating further the ecdysone-independent nature of the receptor-deprivation cell death. In contrast to PG cells, wing disc or salivary glands cells do not require these receptors for survival, revealing their cell and developmental time specificity. Exploring the potential use of this feature of steroid receptors in cancer, we assayed tumor overgrowth induced by altered yorkie signaling. This overgrowth is suppressed by EcR downregulation in PG, but not in wing disc, cells. The mechanism of all these cell death features is based on the transcriptional regulation of reaper. These novel and context-dependent functional properties for ECR and USP receptors may help to understand the heterogeneous responses to steroid-based therapies in human pathologies. Cell Death and Differentiation (2016) 23, 405-416; doi:10.1038/cdd.2015.108; published online 7 August 2015

Steroid hormones control multiple biological processes and, consequently, their faulty regulation underlies many pathologies. $^{1,2}$ The main Drosophila steroid hormone, ecdysone, is synthesized in the larval prothoracic gland (PG) using dietary sterols and cytochrome $P 450$ enzymes. ${ }^{3}$ Following its pulsated secretion into the hemolymph, peripheral tissues convert ecdysone into biologically active 20-hydroxyecdysone (20E) to control larval molting and metamorphosis. ${ }^{4}$

The 20E signal is transduced by heterodimers of two nuclear receptors, ecdysone receptor $(\mathrm{EcR})$ and Ultraspiracle (USP). ${ }^{5}$ USP exhibits a single form throughout development. By contrast, $E c R$ encodes three protein isoforms (EcRA, EcRB1 and EcRB2). These show common DNA- and hormone-binding domains but different $\mathrm{N}$-termini although all of them can heterodimerize USP. ${ }^{6,7}$ Each EcR isoform is hypothesized to have specific functions based on their distinct spatial and temporal patterns and N-termini. ${ }^{8-10} \mathrm{ECR}$ and USP DNA-binding domains recognize ecdysone response sequences which are short palindromes. ${ }^{11}$ Like its vertebrate counterparts, $^{12}$ the ligand-EcR/USP complex activates transcription, whereas the unliganded receptor act as a repressor. $^{13-15}$ The in vivo validation of repressor activities for unliganded complexes, however, is mostly indirect. For example, EcR or USP loss triggers precocious, meaning prior to $20 \mathrm{E}$ surge, cell differentiation through the expression of Broad $Z 1$ in eye disc ${ }^{13}$ or wing sensory neurons. ${ }^{14}$ Therefore, it is assumed that Broad $Z 1$ was repressed by the unliganded EcR/USP. In the activator configuration, the 20E/receptor complex activates characteristic early genes including Broad complex (BrC), E74 and E75. When the corresponding proteins, which are also transcription factors, reach a critical threshold, they repress their own promoters and activate the so called late genes. ${ }^{16,17}$

In humans, estrogen stimulates cell proliferation and survival through activation of the estrogen receptor but, it can also induce tumor regression of hormone-dependent breast cancer in women who have received anti-hormone treatments. ${ }^{18,19}$ The COSMIC data base shows an intriguing upregulation of YAP and TAZ factors in endocrine cancers (thyroid, breast, ovary and prostate). ${ }^{20,21}$ The Drosophila homologue of YAP and TAZ is Yorkie whose overactivation also results in overgrowth. ${ }^{22}$ Cell death during metamorphosis is considered a suitable model to investigate the basic mechanisms of tumor growth regulation by mammalian steroids. However, the puzzling diversity of steroid effects on

\footnotetext{
${ }^{1}$ Department of Molecular, Cellular and Developmental Neurobiology, Instituto Cajal, CSIC, 28002 Madrid, Spain and ${ }^{2}$ Instituto de Biología Evolutiva, CSIC-UPF, 08803 Barcelona, Spain

*Corresponding author: A Ferrús, Department of Molecular, Cellular and Developmental Neurobiology, Instituto Cajal, CSIC, Avenue Dr. Arce 37, 28002 Madrid, Spain. Tel: +34 91585 4739; E-mail: aferrus@cajal.csic.es

${ }^{3}$ These authors contributed equally to this work.

Abbreviations: 20E, 20-hydroxyecdysone; AEL, After egg laying; Atg, Autophagy-related gene; bsk, Basket; Br-C, Broad complex; C3, Caspase-3; COSMIC, Catalogue of Somatic Mutations in Cancer; dIAP, Drosophila inhibitor of apoptosis-1; EcR, Ecdysone receptor; EcRA, Isoform A of the ecdysone receptor; EcRB1, Isoform B1 of the ecdysone receptor; EcRB2, Isoform B2 of the ecdysone receptor; en, Engrailed; FLP, Flipase enzyme; FRT, Flp recombinase target; Gal80 $0^{\text {ts }}$, Gal80 temperature sensitive; Hid, Head involution defective; JNK, Jun-kinase; Igl, Lethal giant larvae; Phm, Phantom; PG, Prothoracic gland; puc, Puckered; ppl, Pump less; RNAi, RNA interference; RT-PCR, Real time polymerase chain reaction; TAZ, Transcriptional coactivator with PDZ-binding motif; USP, Ultraspiracle; wg, Wingless; YAP, Yes-associated protein; yki, Yorkie

Received 09.12.2014; revised 29.6.2015; accepted 01.7.2015; Edited by H Steller; published online 07.8.15
} 
tumor growth, including the lack of effect, challenges the insect approach. In this context, it seems justified to explore cell death mechanisms that could be differentially controlled by steroid receptors versus their canonical hormone ligand, and to assay their effect on Yorkie-dependent tumor outgrowths.

\section{Results}

EcR and USP are required in the PG for metamorphosis. After tool validation (Supplementary Data and Supplementary Figure S1), we analyzed the phenotypes due to depletion of EcRs in the PG by using the phantom (phm-Gal4) driver.

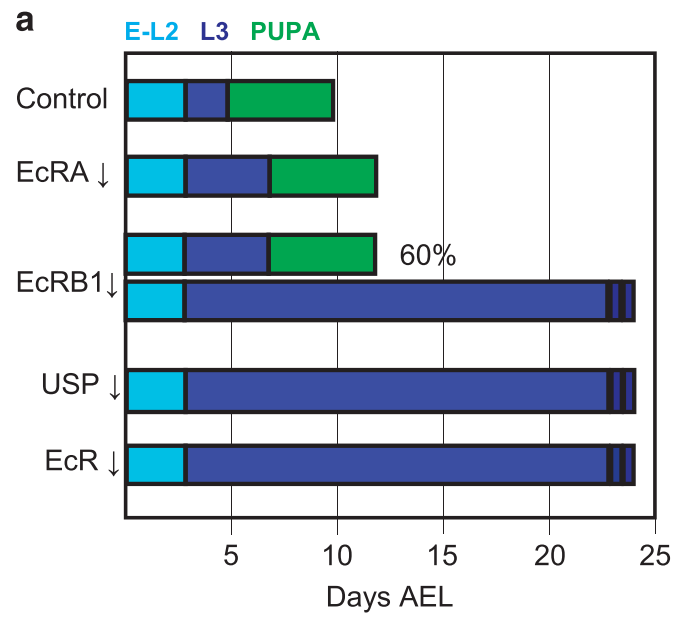

The symbols $\downarrow$ or $\uparrow$ indicate downregulation or upregulation, respectively. The condition EcRA $\downarrow$ allows normal development until L3 stage which is lengthened $48 \mathrm{~h}$ (Figure 1a). The same effect occurs in $60 \%$ of the EcRB1 $\downarrow$ larvae. In the remaining $40 \%$ of EcRB $1 \downarrow$ and $100 \%$ of EcR $\downarrow$ or USP $\downarrow$ larvae, development is halted at L3 stage where they remain over 25 days without entering metamorphosis (Figure 1a) and doubling their size by day 5 past due pupariation time (Figure 1b).

At day 5 post due pupariation time, the fat body of EcR $\downarrow$ and USP $\downarrow$ larvae presents 30\% fewer cells and larger than normal lipid vesicles (Supplementary Figure S3A). Larvae become progressively inactive and develop a brown pigmentation,

b

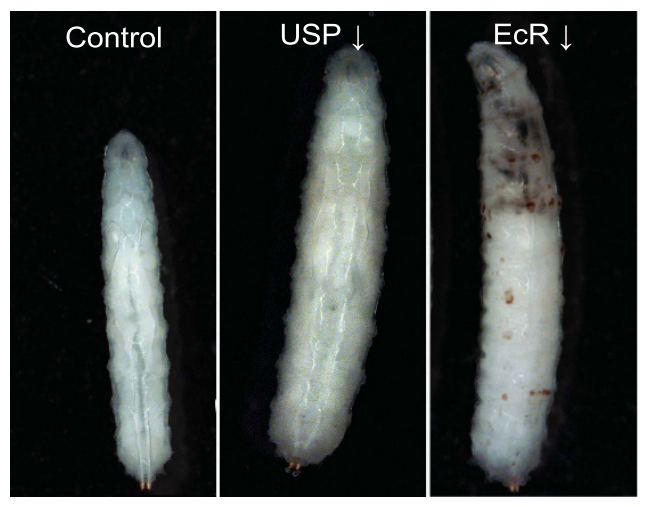

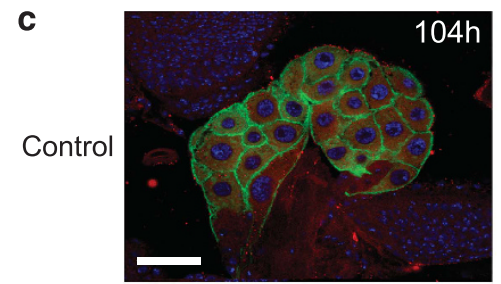
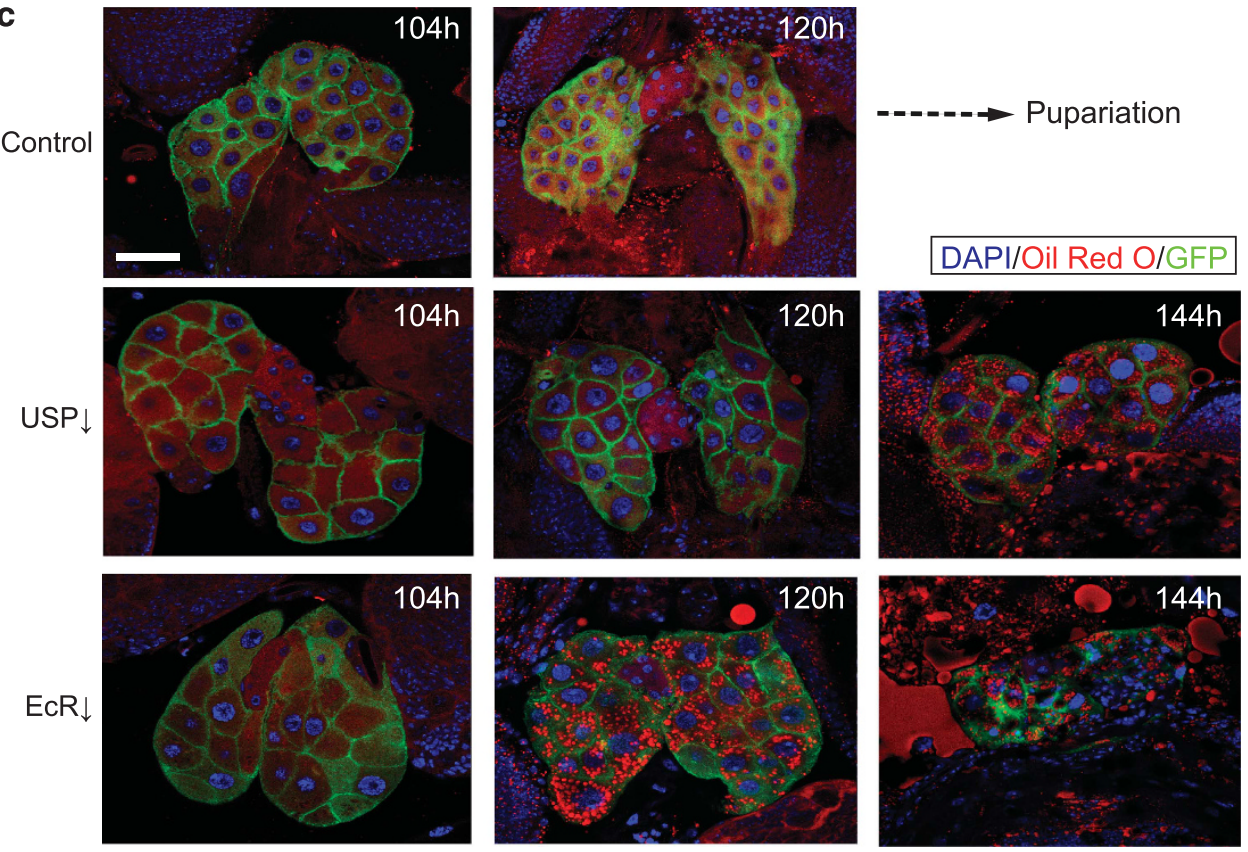

Figure 1 Metamorphosis requires the expression of EcR and USP in the PG. (a) Staged development of genotypes analyzed. phm-Gal4 driven expression of EcR-A ${ }^{\mathrm{RNAi}}$ $(E c R A \downarrow)$ prolongs the duration of $\mathrm{L} 3$; while that of $\mathrm{B} 1$ isoform (EcRB1 $\downarrow$ ) lets $60 \%$ of larvae to pupariate and reach adulthood although with some delay, while about $40 \%$ of them remain as $\mathrm{L} 3$. When all $E c R$ isoforms (EcR $\downarrow)$ or USP (USP $\downarrow$ ) are downregulated, $100 \%$ of larvae never undergo metamorphosis. (b) Representative examples of control late $L 3$ compared with experimental larvae (EcR $\downarrow$ and USP $\downarrow$ ) 5 days past due pupariation time. Notice the larger size of mutant larvae. (c) Larval PGs stained with OilRed-O to reveal lipid content. Control larvae at 104 and $120 \mathrm{~h} \mathrm{AEL}$ show a homogenous and diffuse signal. Under USP $\downarrow$ conditions, lipids accumulate as droplets at $144 \mathrm{~h}$ AEL (24 h delayed with respect to normal). Phenotype is stronger in EcR $\downarrow$ as droplets appear at $120 \mathrm{~h} \mathrm{AEL}$, just before the due pupariation time. Genotypes: Control=phm-Gal4 > UASCD8-GFP; UASLacZ. EcR $\downarrow=p h m-G a l 4>$ UASCD8-GFP; UAS-ECR ${ }^{R N A i}$. USP $\downarrow=p h m-G a l 4>U A S-C D 8-G F P ; ~ U A S-u s p^{R N A i}$. Bar in $\mathbf{c}=20 \mu$ m. Images in $\mathbf{b}$ are at the same magnification 
more prominent in the case of EcR $\downarrow$ than in USP $\downarrow$ (Figure 1b). Although brain size did not appear affected, larval neuromuscular junctions are enlarged with extended branching and double number of synapses (Supplementary Figures S3B and C). This neuronal effect following EcR inactivation in the $P G$ is akin to that previously reported, ${ }^{23}$ albeit, in that report, metamorphosis was delayed, rather than prevented, and the method used was the downregulation of the torso receptor. Moreover, the imaginal discs of larvae with EcR $\downarrow$ and USP $\downarrow$ in the PG show increased cell death and reduced size with abnormal localization of Wingless (Supplementary Figure S3D).

In addition to these systemic effects, the PG was examined in detail. Whereas cell number remained normal, EcR $\downarrow$ and USP $\downarrow$ PGs stained with Oil Red O show lipid droplets abnormally accumulated in the cytoplasm. The accumulation occurred $24 \mathrm{~h}$ sooner in EcR $\downarrow$ animals than in USP $\downarrow$, but in both cases, the effect increased by $144 \mathrm{~h}$ AEL, $24 \mathrm{~h}$ after due pupariation time (Figure 1c).

EcR and USP are required for ecdysone synthesis in PG cells. To study the role of EcR/USP in ecdysone synthesis, we measured the expression of ecdysone-synthesizing genes by qRT-PCR. Expression of disembodied, shadow and phantom increased significantly between early and late L3 stages in controls. However, this was abolished in experimental larvae (Figure $2 \mathrm{a}$ and Supplementary Figures S4A and $\mathrm{C}$ ). The modest increase of torso expression in the control was also absent in experimental larvae (Figure 2a and Supplementary Figure S4D).

To determine whether the failure to pupariate was due to altered levels of ecdysteroids, we measured them. As expected, ecdysteroids increased between early and late control L3 corresponding to the ecdysone synthesis peak previous to pupariation, but that increase was not observed in $\mathrm{EcR} \downarrow$ or USP $\downarrow$ larva (Figure $2 \mathrm{~b}$ ). When ecdysone production is compromised, metamorphosis can be rescued by feeding $20 \mathrm{E}$ to mutant larvae. Surprisingly, this procedure failed in our $\mathrm{EcR} \downarrow$ and USP $\downarrow$ larvae, although it succeeded to rescue phmGal4 > UAS-phm ${ }^{R N A i}$ larvae (data not shown).

Inspection of phm-Gal4 EcR $\downarrow$ and USP $\downarrow$ larvae showed a weak GFP reporter signal in the trachea that increased with age (Supplementary Figure S5). This signal prompted a closer analysis of the GFP expression domain in phm-Gal4> UASGFP control larvae. The reporter was already detected in controls albeit at a much lower intensity. Thus, the trachea

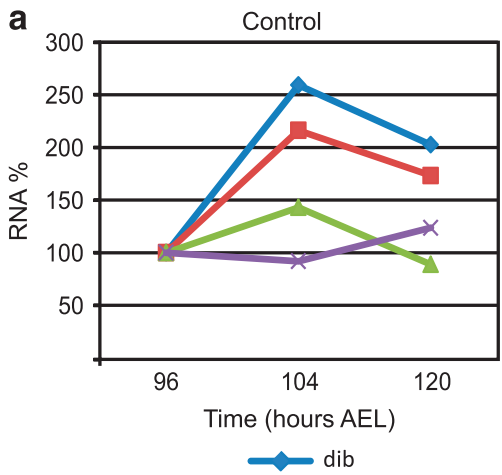

b

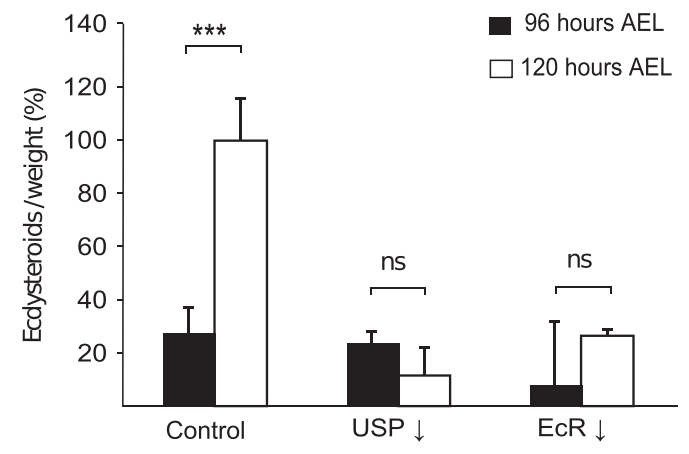

USP $\downarrow$

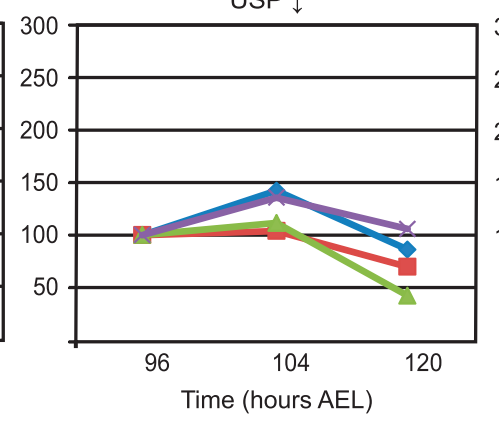

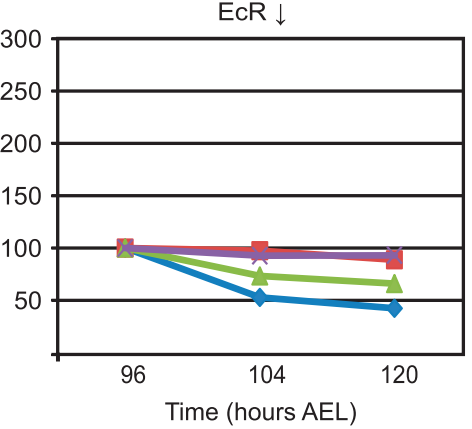

$\therefore$ torso
C
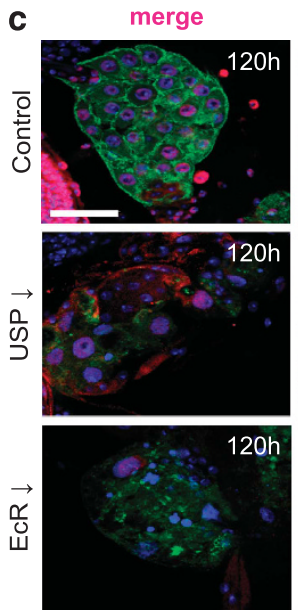

Broad

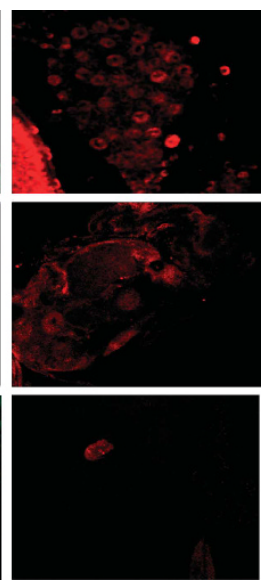

Figure 2 EcR and USP are required for ecdysone synthesis. (a) Transcriptional profiles for three ecdysone-synthesizing genes (dib, disembodied; sad, shadow and phm, phantom) and the PTTH receptor torso. The qRT-PCR data from whole larvae were obtained at 96,104 and $120 \mathrm{~h}$ AEL, and are shown as a percentage of the value at $96 \mathrm{~h} \mathrm{AEL}$. In the control, gene expression peaks just before puparium formation, while in USP $\downarrow$ or EcR $\downarrow$ larvae, this peak does not occur. Triplicate measurements from experiments repeated three times. Graphs containing error bars and significance for each gene are shown in Supplementary Figures S4 A-D. (b) Ecdysteroid (E and 20E) levels in control and mutant larvae at different time points. Data $(\mathrm{pg} / \mathrm{mg})$ are normalized to the $120 \mathrm{~h}$ time point. The peak of ecdysteroids that triggers pupariation in control larvae is not detected in larvae expressing EcR $\downarrow$ or USP $\downarrow$ in the PG. (c) Broad expression (red) in control PG is substantially abolished by USP $\downarrow$ or EcR $\downarrow$ at $120 \mathrm{~h}$. Genotypes: Control $=$ phmGal4 > UAS-CD8-GFP; UAS-LacZ. EcR $\downarrow=p h m-G a l 4>$ UAS-CD8-GFP; UAS-EcR ${ }^{R N A i}$. USP $\downarrow=p h m-G a l 4>$ UAS-CD8-GFP; UAS-USp ${ }^{R N A i}$. Bar $=20 \mu m$ 
should be included jointly with the wing disc (see below) as a low intensity expression domain of phm-Gal4. Likely, the overdue experimental larvae increase $\mathrm{phm}$-Gal4 expression in these low intensity domains. The cumulative expression will increase also the depletion of $20 \mathrm{E}$ receptors in target tissues rendering them unable to respond to the fed $20 \mathrm{E}$ and, consequently, larvae do not enter metamorphosis.

As further evidence that EcR $\downarrow$ and USP $\downarrow$ PGs do not produce ecdysone signaling, we monitored $\mathrm{Br}-\mathrm{C}$ expression. This family of zinc finger transcription factors is upregulated in all cell types as an early response to the ecdysone surge of late L3. ${ }^{24}$ The data show that $\mathrm{Br}-\mathrm{C}$ is not detected in experimental PGs (Figure 2c). Thus, we conclude that the characteristic function of PG cells, pro-hormone synthesis, requires $\mathrm{ECR}$ and USP.

EcR and USP, but not ecdysone, deprivation affects PG cell survival. The functional defects of $P G$ cells prompted an analysis of cell survival, either causative of, or resulting from, EcR/USP downregulation. Activated caspase-3 (C3) served as cell death indicator. Control larvae do not show immunoC3 staining. However, EcR $\downarrow$ and USP $\downarrow$ glands show consistent signs of cell death which become massive by $144 \mathrm{~h}$ (Figure 3a). Many C3-positive cells also show fragmented nuclear bodies revealed by DAPI. The TUNEL assay confirmed the apoptosis-like process of $P G$ cell death under EcR $\downarrow$ or USP $\downarrow$ conditions (Figure 3b).

As ecdysone synthesis failure and cell death coincide in time, we questioned whether ecdysone deprivation could cause cell death. To that end, we analyzed PGs where phantom, one of the ecdysone-synthesizing genes, is knocked down. In this experiment, the phantom $\downarrow$ condition was switched-on in L2, using the Gal80 ${ }^{\text {ts }}$ repressor, in order to bypass the vital requirement of this gene in embryogenesis. As expected, these larvae showed extremely low ecdysteroid levels (Supplementary Figure S6) and did not pupariate. However, opposite to EcR $\downarrow$ or USP $\downarrow$, phantom-depleted PG cells did not show C3 activation (Figures $3 c$ and d). Actually, cells showed a notable increase in size. Next, we questioned whether ecdysone could be required for PG cell survival through an autocrine mechanism. To that end, we inactivated Kish and Gryzun, two genes required for vesicle trafficking and secretion. ${ }^{25}$ In both cases, PG cells did not show signs of apoptosis although, as expected, larvae did not pupariate (Figures $3 e$ and f). We conclude that the lack of ecdysone does not cause PG cell death. More likely, the activation of a cell death program may cause halting of ecdysone synthesis.

EcR- and USP-depleted PGs degenerate through a mechanism different from autophagy. Wild-type $P G$ degenerates during metamorphosis in a process that shows electron microscopy features characteristic of autophagy, rather than apoptosis. ${ }^{26}$ To ascertain the mechanism of PG degeneration in the absence of EcR or USP, we considered autophagy. The Atg8-cherry marker appears to indicate autophagy triggering because the reporter signal accumulated in cytoplasmic dots, presumably autophagosomes (Figure 4a). However, downregulation assays of autophagy genes, ${ }^{27}$ including Atg1, Atg4, Atg5 and Atg7 were unable to rescue $\mathrm{EcR} / \mathrm{USP}$-dependent cell death in the $\mathrm{PG}$ or the failed pupariation phenotype (Figure 4b). To consider alternative cell death mechanisms, we screened through genetic modifiers. The dIAP1 $\uparrow$ and reaper $\downarrow$ conditions were most effective, along with constitutively active ras $\uparrow$ and $p 35 \uparrow$ (a caspase inhibitor from baculovirus). Notably, dIAP1 $\uparrow$ was a more effective suppressor of EcR $\downarrow$ than of USP $\downarrow$ (Figure 4b). This differential effect could reflect the distinct set of genes targeted by EcR versus USP. On the other hand, sickle $\downarrow$ and hid $\downarrow$ (apoptosis effectors) failed to rescue the L3 arrest phenotype. In addition, upregulating the receptor that triggers ecdysone synthesis, Torso, ${ }^{28}$ or upregulating the early effectors of ecdysone signaling, $B r-C$ genes, ${ }^{24}$ also failed to bypass the deleterious EcR or USP downregulation (Figure 4b). This is consistent with the lack of ecdysteroid synthesis in experimental larvae shown by direct measurements above (Figure $2 b$ ).

Interestingly, mutated EcR isoforms that do not bind ecdysone, EcRB1 ${ }^{\mathrm{W} 650 \mathrm{~A}}$, or cannot activate target gene transcription, EcRB1 ${ }^{\mathrm{F} 645 \mathrm{~A}} 7$ rescue to some extent the metamorphosis blockade (Figure 4b). This is additional evidence that EcR/USP-dependent effects in the $P G$ are ecdysone-independent. Further, downregulating Eip93F, which is a cell death signal induced by the pupal ecdysone pulse once metamorphosis is underway and that regulates reaper and other apoptosis genes during salivary gland cell death, ${ }^{29}$ failed to rescue the phenotype under study (Figure 4b). Thus, Eip93F can also be excluded from the EcR/USP-dependent cell death mechanism.

Beyond the use of RNAi lines to elicit the USP/EcR $\downarrow$ conditions, we tested also FRT/FLP clones of mutant allele $u_{s p} p^{5}$ (Figures 4c and d). PG cells show smaller than normal and fragmented nuclei (Figure $4 \mathrm{c}$ ) but the co-expression of EcRA rescues that phenotype (Figure 4d). Finally, the downregulation of EcR or USP increases reaper transcription (Figure 4e, left panel), and a concomitant decrease in dIAP1, a prosurvival gene (Supplementary Figure S4E). Together, these data support the cell survival requirement of EcR and USP in the PG and suggest that their deprivation causes cell death through a mechanism that is unrelated to the autophagy that occurs during pupal metamorphosis, but that is similar to apoptosis. ${ }^{30}$ In this context, then, it is likely that the observed activation of Atg8-cherry, which is not followed by the involvement of autophagy genes, could reflect a stressdependent event as previously described. ${ }^{31}$

\section{EcR and USP promote PG cell survival by repressing the} JNK pathway. The Jun Kinase (JNK) pathway activates apoptosis in many Drosophila cells where the enzyme is encoded by basket (bsk). ${ }^{32,33}$ We explored the JNK signaling in the PG cell death induced by EcR or USP depletion. To that end, we monitored the expression of its effector puckered (puc) as reported by puc-LacZ. While control PG does not show noticeable puc-LacZ expression, the EcR $\downarrow$ condition does (Figures $5 \mathrm{a}$ and $\mathrm{b}$ ). The puc-LacZ signal was validated by direct bsk overexpression in the $\mathrm{PG}^{34}$ (Figure $5 c$ ). Driving bsk causes PG cell death with caspase-3 activation (Figure 5d) and, as expected, failure to enter metamorphosis. Interestingly, the co-expression of EcRB1 ${ }^{\text {W650A }}$ suppresses the bsk/JNK effect (Figure 5e) and allows larvae to pupariate and reach adulthood. This suppression includes the 


\section{a}
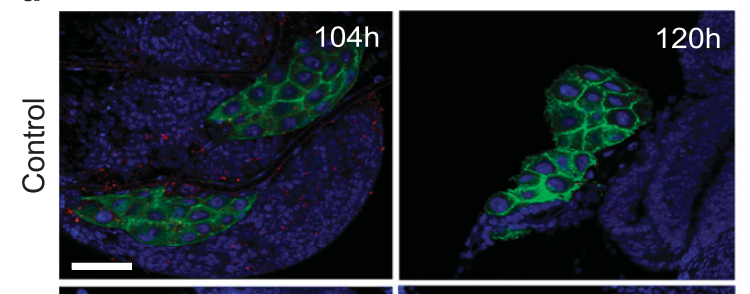

-1 Pupariation
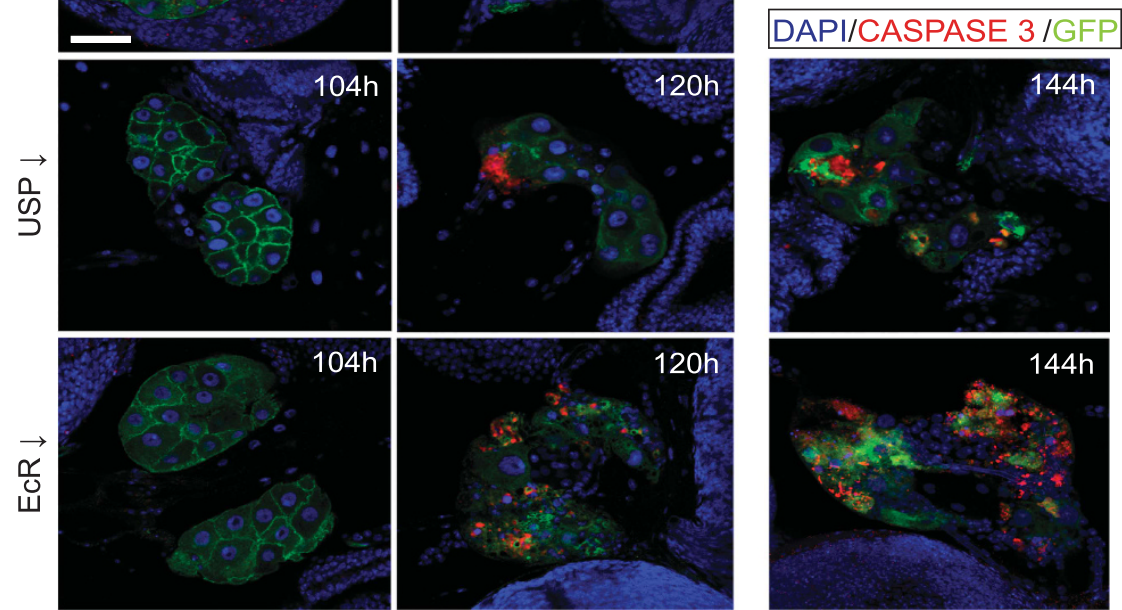

b

Control

USP $\downarrow$
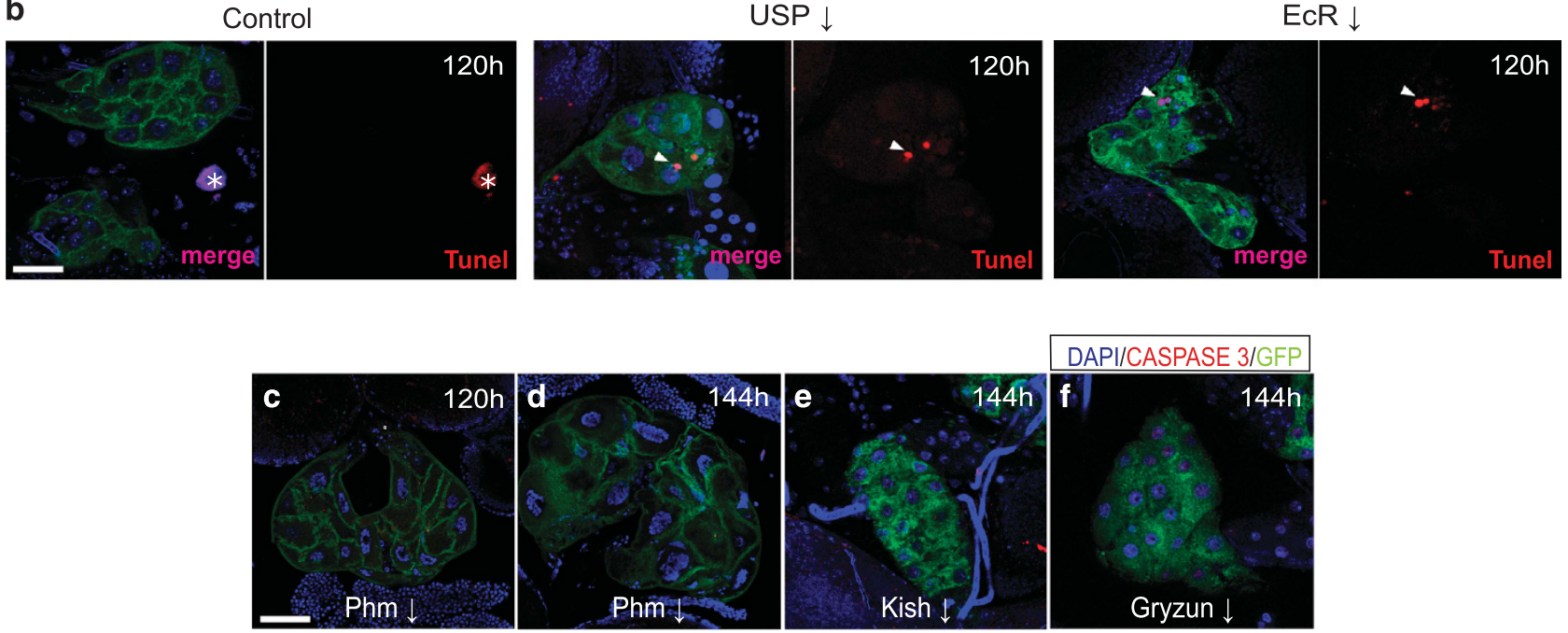

DAPI/CASPASE 3/GFP

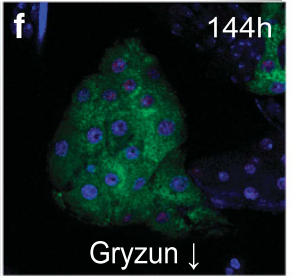

Figure 3 Survival of PG cells requires EcR and USP, but not ecdysone. (a) In control PG, cells do not show activated caspase-3 (C3) (red). However, under EcR $\downarrow$ or USP $\downarrow$ conditions, many cells become C3-positive by the normal pupation time (120 h), and their number increases by $144 \mathrm{~h}$. (b) TUNEL assay (arrow heads) detects apoptosis in EcR $\downarrow$ and USP $\downarrow$, but not in control PG cells at $120 \mathrm{~h}$. The asterisk marks a TUNEL-positive cell outside the PG and serves as internal control of the assay. (c) Downregulation of phantom in L2 PG prevents cells from initiating metamorphosis and synthesize ecdysone (see Supplementary Figure S6). However, instead of activating apoptosis, cells greatly enlarge by $120 \mathrm{~h} \mathrm{(c)} \mathrm{and} \mathrm{more} \mathrm{so} \mathrm{by} 144 \mathrm{~h}$ (d). (e, f) Blocking ecdysone secretion by downregulating Kish (e) or Gryzun (f) does not elicit caspase-3-dependent apoptosis although larvae do not enter into metamorphosis. Genotypes: Control=phm-Gal4> UAS-CD8-GFP; UAS-LacZ. EcR $\downarrow=$ phm-Gal4>UAS-CD8-GFP; UAS-ECR ${ }^{R N A i}$.

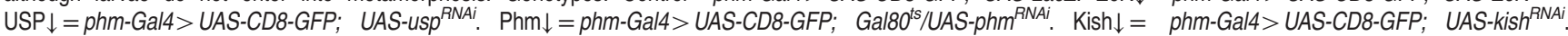
Gryzun $\downarrow=$ phm-Gal4 $>$ UAS-CD8-GFP; UAS-gry ${ }^{R N A i}$. Bar $=20 \mu \mathrm{m}$

transcriptional normalization of reaper (Figure 5f), an apoptosis hallmark gene whose transcription is directly regulated by EcR/USP. ${ }^{35}$ As expected, dIAP1 transcription is reduced by bsk/JNK $\uparrow$ and normalized to some extent by EcRB1 ${ }^{\text {W650A }}$ (Supplementary Figure S4F). These data favor an apoptosis-type mechanism for the EcR/USPdependent cell death in the PG. It is plausible that EcR/ USP depletion triggers JNK activation through puc and, simultaneously, relieves repression of reaper. This relief would further activate JNK which, in turn, would induce reaper expression. On the other hand, the suppression of apoptotic features by EcRB1 ${ }^{\mathrm{W} 650 \mathrm{~A}}$ overexpression further supports the ligand-independent nature of the EcR requirement for cell viability.

Imaginal disc and salivary gland cells do not require EcR/USP for cell survival. To test whether the effects of EcR or USP depletion in the PG are common to other cell 
a
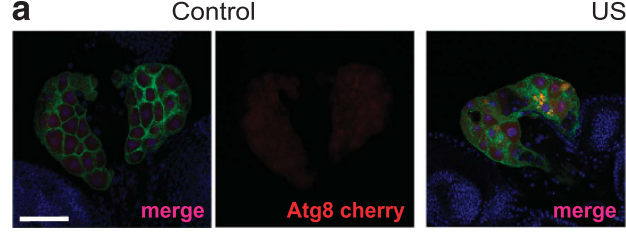

USP $\downarrow$

b

NON RESCUE:

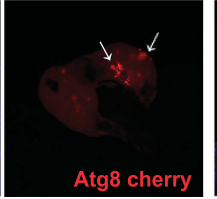

$\mathrm{EcR} \downarrow$

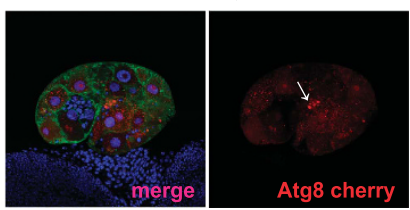

Torso $\uparrow$; USP

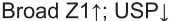

Broad Z2 $\uparrow ;$ USP $\downarrow$

Broad Z3 $\uparrow ; \mathrm{EcR} \downarrow$

Broad Z4 个; EcR $\downarrow$

Hid $\downarrow ;$ USP $\downarrow$

Sickle $\downarrow ;$ USP $\downarrow$

Eip93F $\downarrow ;$ USP $\downarrow$

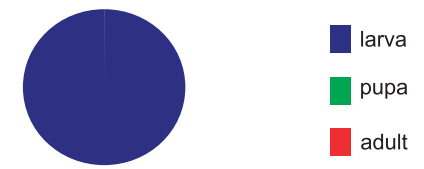

RESCUE:
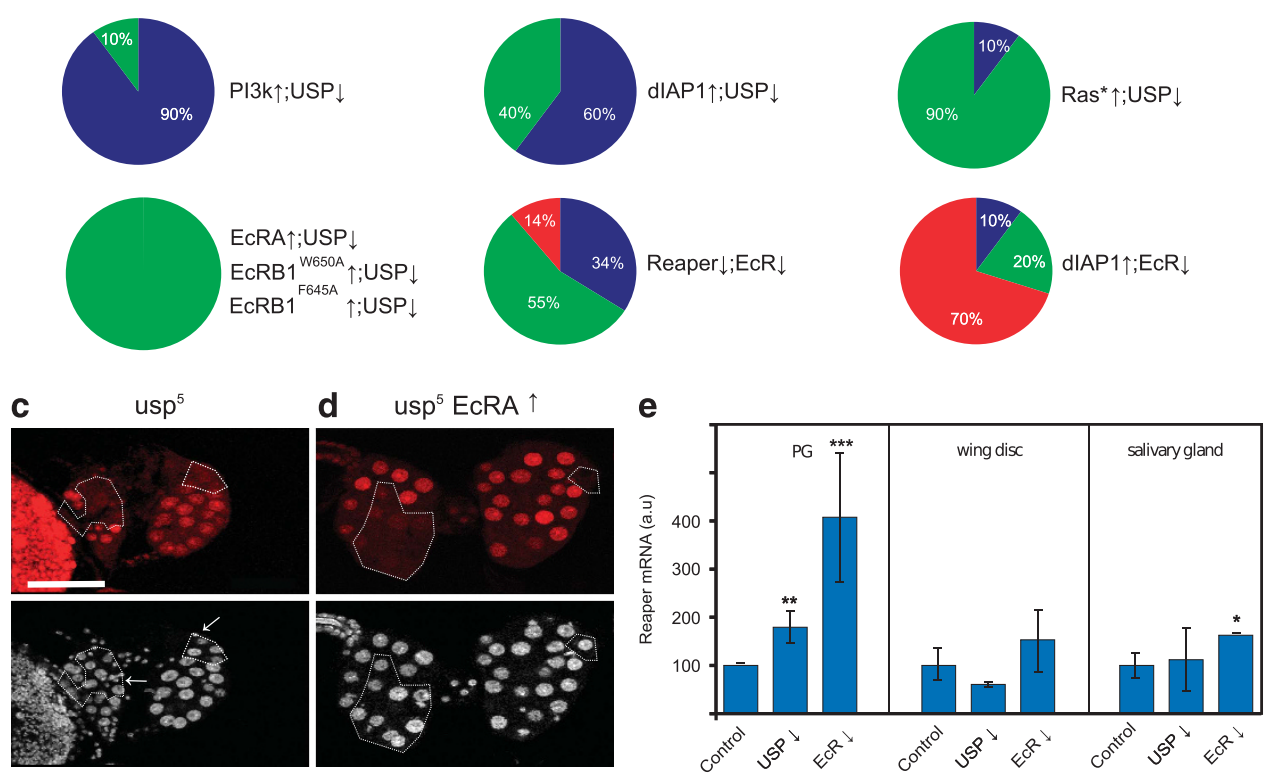

Figure $4 \mathrm{EcR}$ and USP phenotypes are rescued by downregulating apoptosis genes. (a) The cherry-reported autophagy marker Atg8 accumulates (mainly in autolysosomes) of PG cells when USP or EcR are knocked down (arrows in right panels) compared with the background signal from control PG (left panel). This could suggest that autophagy has been initiated in the absence of EcR or USP. (b) Attempts to rescue the metamorphosis phenotypes due to USP $\downarrow$ or EcR $\downarrow$. Circles indicate developmental stages $($ larva $=$ blue, pupa $=$ green, adult $=$ red) and numbers indicate the relative proportion of each stage $(n>100)$. Note that apoptosis genes (e.g.: dlAP1 or reaper) rescue the mutant phenotypes to various extents. Autophagy genes (Atg), however, do not. Also, regular targets of ecdysone signaling (e.g.: Broad or Eip93F) are unable to rescue. (c, d) FLP/FRT clones (absence of red signal, dotted lines) in the PG. usp 5 clones (c) show small cells with fragmented nuclei (arrows), while the simultaneous upregulation of EcRA (d) rescues that phenotype. Only five-stack maximum projections are shown for clarity. (e) Reaper expression levels by qRT-PCR in dissected Ring glands (phm-Gal4), wing discs and salivary glands (rotund-Ga/4) at $120 \mathrm{~h}$. Only in the PG, there is a strong upregulation of reaper in the absence of EcR or USP which illustrates the differential cell

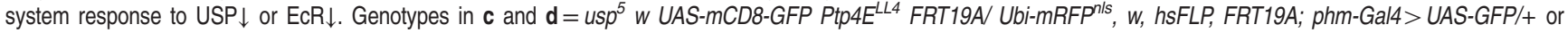
UAS-ECRA, respectively

types, we analyzed the wing pouch and salivary glands using the rotund-Gal4 driver combined with the elav-Gal80 repressor. Suppressing the driver in the nervous system was needed to allow larval viability. Following C3 immunostaining, no increase of apoptosis was detected in either cell system (Figures $6 \mathrm{a}$ and $\mathrm{h}$ ). Also, bsk $\uparrow$-induced apoptosis in the wing pouch was not rescued by upregulating $\mathrm{EcR}$ isoforms, including the one that does not bind ecdysone, in contrast to PGs (Figures $5 \mathrm{~g}$ and j). These data are consistent with the lack of reaper upregulation in wing discs and salivary glands in EcR $\downarrow$ and USP $\downarrow$ animals (Figure $4 \mathrm{e}$, right panels). The absence of cell survival effects was also confirmed in the leg disc (en-Ga/4) and Malpighian tubuli (ppl-Ga/4) by the C3 criterion
(Supplementary Figure S7). Also, depletion of EcR or USP in the wing pouch did not affect cell proliferation as revealed by the phospho-histone $\mathrm{H} 3$ criterion (Supplementary Figure S8).

In the salivary glands, cell size was strongly reduced in USP $\downarrow$ and $E c R \downarrow$ conditions (Figures $6 e$ and i). This phenotype was not rescued by upregulating any EcR isoform, in particular $\mathrm{EcRB}^{\mathrm{W} 650 \mathrm{~A}}$, indicating that it could be ecdysone-dependent. A minor increment of reaper expression was detected with EcR $\downarrow$ (Figure 4e), suggesting that depletion of this receptor, but not USP, could have some deleterious effect on salivary gland cells although still compatible with viability. Thus, we conclude that EcR and USP are required for cell survival in a cell type-specific manner. 

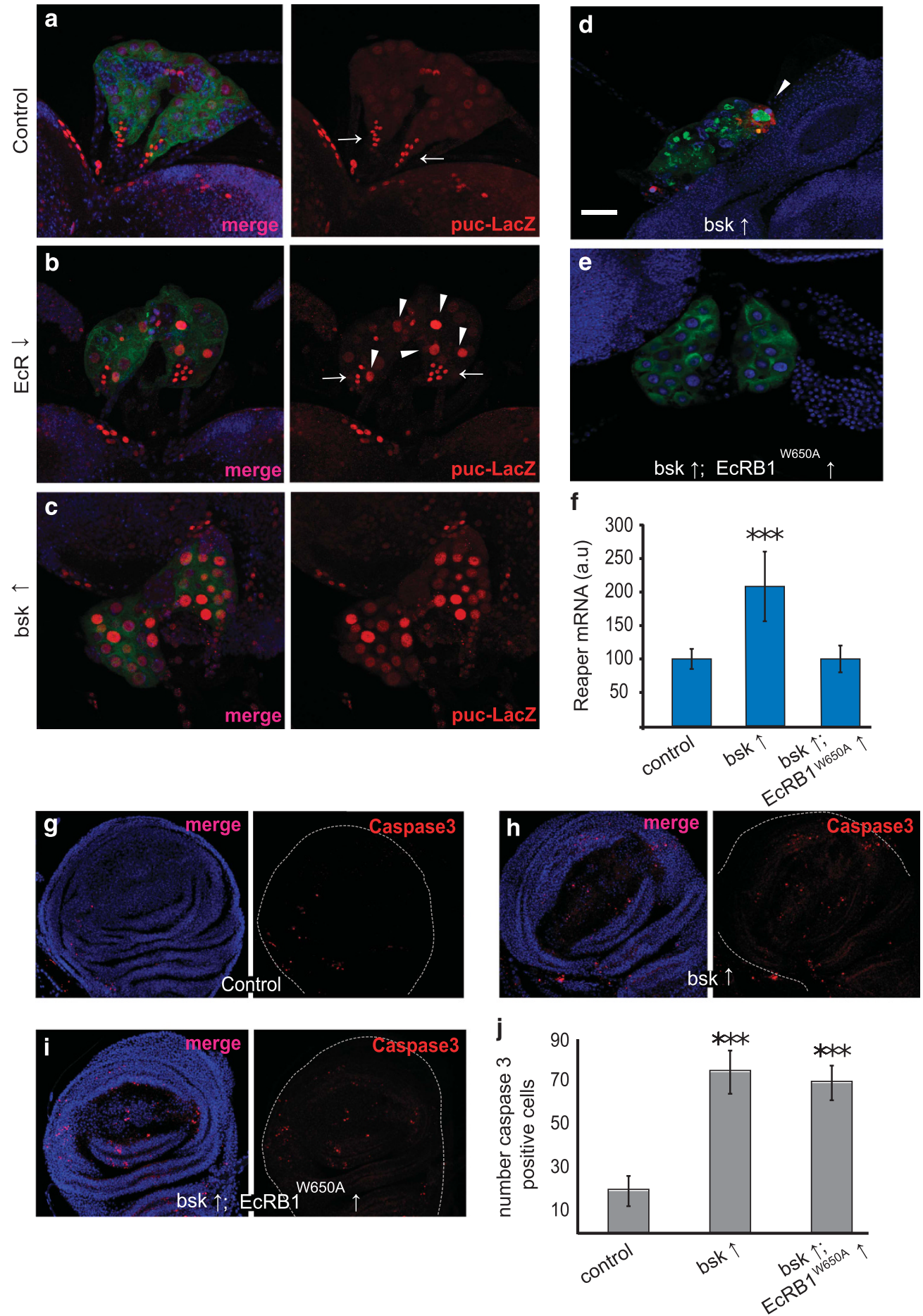

Figure 5 EcR-related cell death in the PG activates Basket/JNK. (a) Control PG cells (120 h) do not activate the bsk-encoded JNK as monitored by the LacZ-reported expression of puckered (puc). Notice the puc-LacZ expression (red) in some cells of the brain and Ring Gland (arrows), but not in those of the PG. (b) Following the driven EcR downregulation at $96 \mathrm{~h}$, however, puc-LacZ becomes upregulated (arrow heads) by $120 \mathrm{~h}$. (c) As expected, the direct upregulation of bsk in the PG at $96 \mathrm{~h}$ reflects into the expression increase of puc-LacZ as visualized at $120 \mathrm{~h}$. (d-f) bsk overexpression in PG induces apoptosis by $144 \mathrm{~h}$ (d), as indicated by activated caspase-3 (arrow head), and larvae do not pupate, akin to $E c R \downarrow$ or USP $\downarrow$ conditions (see Figure 1), but the phenotype is rescued by $E c R-B 1^{W 650 A} \uparrow(e)$ which allows larvae to pupariate and yield normal adults. This suppression includes the transcriptional normalization of reaper expression (f) as measured by qRT-PCR in dissected Ring glands. (g-i) Caspase-3 monitored apoptosis (red) in the wing disks (rotund-Ga/4) of control (g) or bsk (h) larvae are not rescued by EcR-B1 ${ }^{\text {W650A }} \uparrow$ (i). (j) Quantification of caspase-3-positive wing disc cells in the genotypes

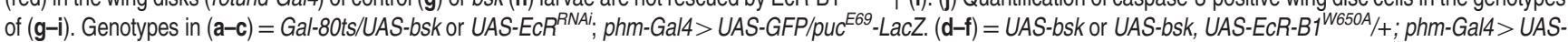
GFP/+. $(\mathbf{g}-\mathbf{j})=r n-G a l 4>$ UAS-bsk or UAS-bsk, UAS-EcR-B1 ${ }^{\text {W650A }}$. Bar in $\mathbf{d}=20 \mu \mathrm{m}$ 

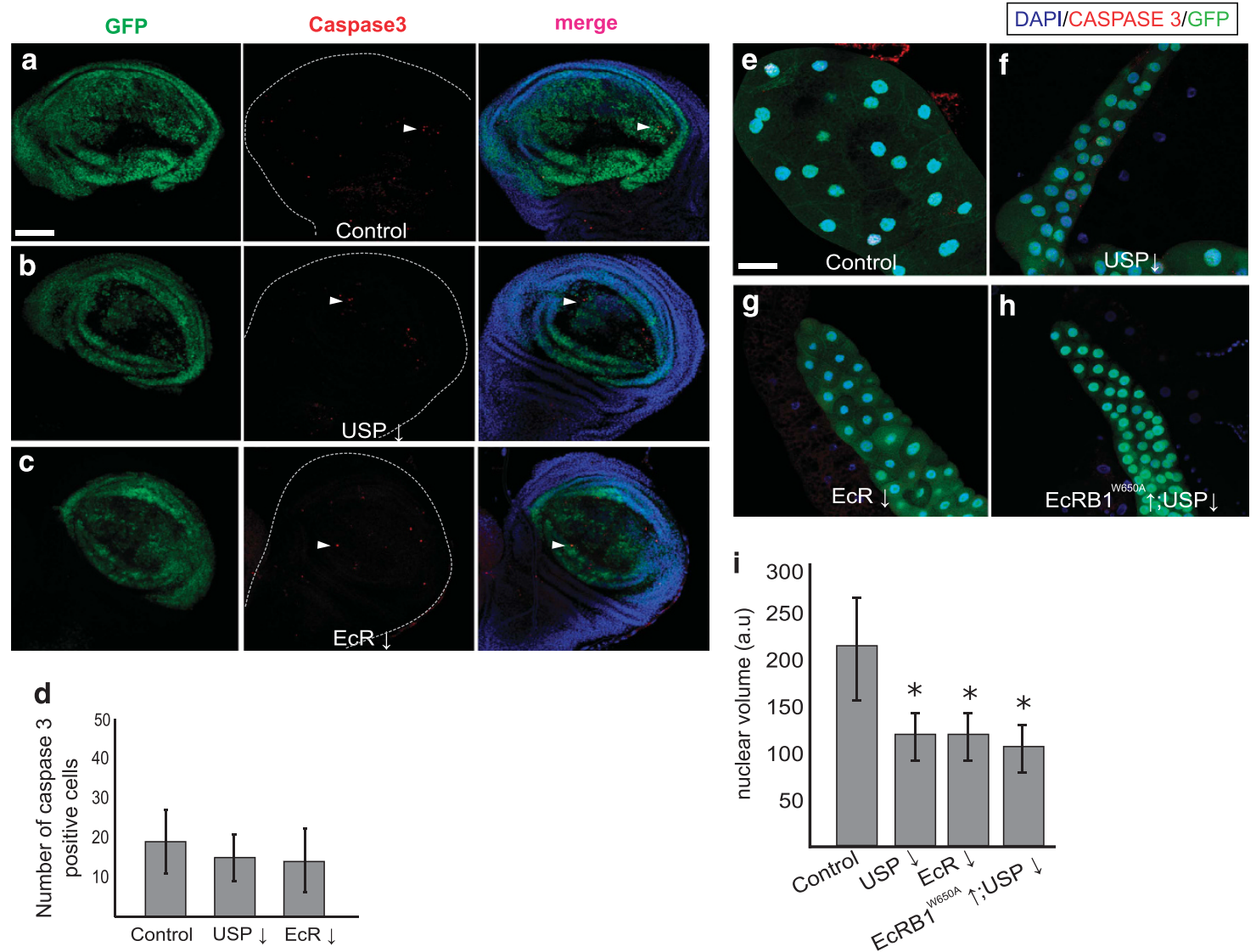

Figure 6 Cell type-specific responses to EcR or USP downregulation. Staining for activated caspase-3 (red, arrow head) in rotund-Gal4 (green) wing discs from control (a), USP $\downarrow$ (b) or EcR $\downarrow$ (c) genotypes. (d) Quantification of cell death showed no difference for all genotypes. (e-h) Experimental salivary glands (rotund-Gal4) showing caspase-3 in control $(\mathbf{e}), \mathrm{USP} \downarrow(\mathbf{f}), \mathrm{EcR} \downarrow(\mathbf{g})$ or EcR-B1 ${ }^{\mathrm{W6} 50 \mathrm{~A}} \uparrow \mathrm{USP} \downarrow(\mathbf{h})$ genotypes. No evidence of apoptosis is detected. However, nuclear size is reduced in USP $\downarrow$ or EcR $\downarrow$ and the phenotype is not rescued by EcR-B1 ${ }^{\text {W650A }} \uparrow$ (h). (i) Salivary gland nuclear volumes from e-h genotypes. Genotypes $=r n-G a l 4>U A S-u S p^{R N A i}(\mathbf{b}, \mathbf{f})$ or $U A S-E c R^{R N A i}(\mathbf{c}, \mathbf{g})$ or UAS-USp ${ }^{R N A i}, U A S-E C R-B 1^{W 650 A}$ (h). Bar in a and $\mathbf{e}=20 \mu \mathrm{m}$

EcR and USP can repress tumor growth. Anti-hormonal treatment is often used against human cancers that overexpress steroid receptors. However, development of resistance and heterogeneous responses to steroid treatments over prolonged therapy are not uncommon. ${ }^{36,37}$ We reasoned that the ligand-independent cell survival effects of EcR and USP in the PG of Drosophila could eventually help to understand that heterogeneity of responses to therapies. In that context, we explored the effects of EcR and USP deprivation in yorkie-elicited PG tumors. A constitutively active form of $y k i, y k i^{*}$, (see Materials and Methods) was driven to the PG yielding a substantial overgrowth (Figures 7a and b). The overgrowth was largely suppressed by EcR $\downarrow$ (Figure $7 \mathrm{c}$ ) and about $10 \%$ of larvae entered metamorphosis. Larvae that did not pupariate showed activated C3 staining over time (168 h AEL) (Figure 7d). The $y k i^{*}$-induced overgrowth during $L 3$ could be restrained also by bsk-induced cell death, albeit, in this case, no larvae entered metamorphosis and overdue PGs (168 h AEL) showed abundant C3 activity (Figures $7 e$ and f). Thus, EcR $\downarrow$ rather than bsk $\uparrow$, seems a better strategy against this type of tumors.

Although seldom mentioned, the phm-Gal4 expression domain includes a low intensity, small number of wing disc cells corresponding to the adult wing hinge (Figure $7 \mathrm{~g}$ ).
We took advantage of this domain to analyze $y k i^{*}$ and $E c R$ effects in the wing disc to test the cell type specificity of EcR requirement in the context of tumor overgrowth. $y k^{*}$ caused overgrowth by $120 \mathrm{~h} \mathrm{AEL}$ (Figure 7h) that, contrary to the PG, neither EcR $\downarrow$ (Figure 7i) nor bsk $\uparrow$ (Figure 7j) conditions could counteract. Past the due pupariation time (168 h AEL), the $y k{ }^{*}$-induced overgrowth became massive and, again, neither of the two experimental conditions repressed it (Figures $7 \mathrm{k}$ and I). The overgrown domain of wing discs expressed wingless $(w g)$ albeit in an aberrant pattern (Supplementary Figure S9).

We addressed the mechanism of EcR $\downarrow$-dependent cell death in the context of $y k i^{*}$-dependent tumor condition in the PG. As $y k i$ is a reaper repressor, ${ }^{38}$ we monitored by qRT-PCR the expression levels of reaper in dissected Ring glands (Figure $7 \mathrm{~m}$ ). The data show a significant transcription increase by either of the two methods of inducing cell death, bsk $\uparrow$ or EcR $\downarrow$, even when overexpressing $y k^{*}$. To discard that the partial suppression of tumor overgrowths could result from using several UAS target constructs that may have diluted the Gal4 driver, we generated genotypes with an extra UAS-cherry construct. Under these conditions, no weakening of $b s k$ or $y k i^{*}$ phenotypes in the PG were evident (Supplementary Figure S10). 

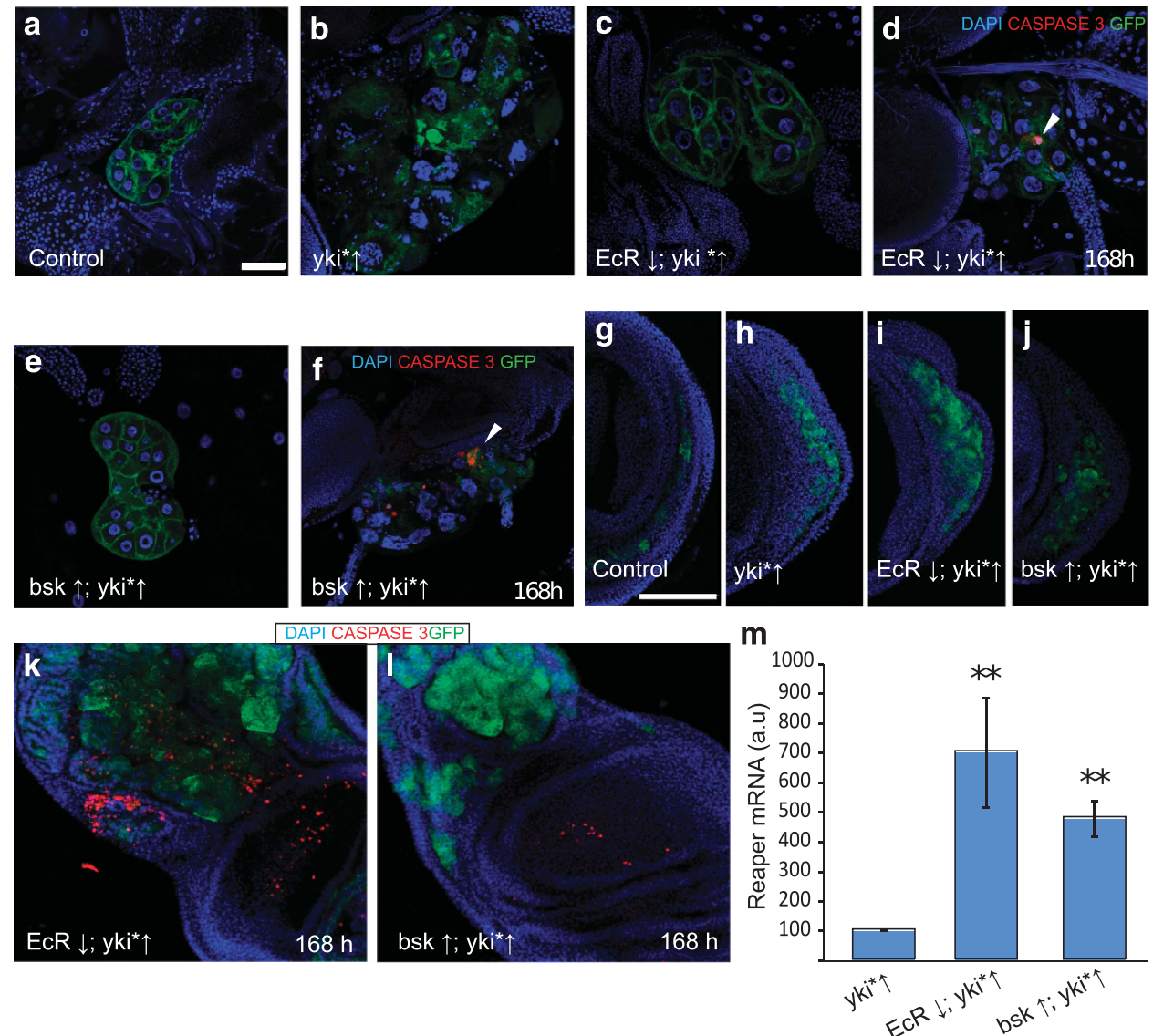

Figure 7 EcR counteracts tumor overgrowth in PG, but not wing disc, cells. (a-f) Normal and experimental PG at two developmental times, $120 \mathrm{~h}$ (a-c, e) and $168 \mathrm{~h}$ (d, f). Caspase-3-positive cells are in red (arrow head). ( $(\mathbf{g}-\mathbf{l})$ Normal and experimental wing discs at the same developmental times. Note that caspase-3-positive cells are present in the wing tissue but not in the tumor overgrowth. $Y k^{\star}{ }^{\star}$ and bsk overexpression causes cell death (red) within the overgrowth. However, this cell death does not counteract the proliferation rate in the tumor, and the wing disc develops a very large hyperplasia by several days after the due pupariation time (I). (m) qRT-PCR data of reaper expression in dissected Ring glands of the tumor-inducing genotypes. Note the strong upregulation of reaper. Genotypes: phm-Gal4> UAS-CD8-GFP; UAS-LacZ (control) (a, g), UAS$y k^{S 111 A, S 168 A, S 250 A}\left(y k^{*}\right)(\mathbf{b}, \mathbf{h}), U A S-E C R^{R N A i}(\mathbf{c}, \mathbf{d}, \mathbf{i}, \mathbf{k})$ or UAS-bsk $(\mathbf{e}, \mathbf{f}, \mathbf{j}, \mathbf{l})$. Bar in a and $\mathbf{g}=20 \mu \mathrm{m}$

Finally, in an attempt to explore other tumor-eliciting conditions, we generated FLP/FRT somatic clones for the double condition $\operatorname{Ras}^{V 12} / /\left.g\right|^{R N A i}$ and tested the effect of USP $\downarrow$. The simultaneous attenuation of lethal-giant-larvae ( $\mid g l)$ and the constitutive activation of $\operatorname{Ras}\left(\operatorname{Ras}^{V 12}\right)$ yield enlarged PG cells, albeit not tumorous overgrowths (Supplementary Figure S11A). However, USP $\downarrow$ did rescue this phenotype (Supplementary Figure $\mathrm{S} 11 \mathrm{~B}$ and $\mathrm{C}$ ) which validates the USP/EcR effects on overgrowths of a different origin.

\section{Discussion}

The data show a novel requirement for steroid receptors EcR and USP for cell survival that is ligand-independent and cell type-specific. PG cells depend on these receptors and their deprivation causes apoptosis-like death that involves reaper, $b s k / J N K$ and $d I A P$. This death process seems unrelated to autophagy which characterizes the ecdysone-triggered cell degradation of other larval tissues at normal metamorphosis. The case further illustrates the diversity of pathways, often sharing signals, which can lead to cell death. Recently, evidences have been gathered indicating that sensitivity to apoptosis is acquired by tissues at two specific steps during development, embryogenesis and pupariation. ${ }^{39}$ The data on EcR and USP coincide with the pupariation time requirement and provide a mechanism for this sensitivity.

On the basis of phm-Gal4 and phm-Gal4/Gal80 ds driving systems, two types of experiments have been carried out: chronic and L2 switch-on ( $\left.\mathrm{Gal}-80^{t s}\right)$, respectively. The EcR $\downarrow$ and USP $\downarrow$ conditions were tested in both experiments yielding the same result, arrest in late L3 before pupariation. This, plus the lack of detectable ecdysteroid biosynthesis, frames the temporal requirement of EcR and USP for cell survival just prior to metamorphosis at $120 \mathrm{~h} \mathrm{AEL}$. The phm-Gal4 driver is expressed in early embryo. ${ }^{40}$ However, the two receptors do not appear to cause deleterious effects, further supporting the pre-metamorphosis time requirement for cell survival. By contrast, experiments with bsk and $\mathrm{Br}-\mathrm{C}$ had to be carried out in the L2 switch-on mode to bypass their early lethality.

From this study, it seems clear that liganded and unliganded EcRs must either regulate different gene repertoires or operate in different modes on the same gene repertoire. As a conceptual frame, two types of ecdysone response sequences in the target genes have been proposed: ${ }^{14}$ 
inductive ones, where the liganded receptor strongly activates transcription; and permissive ones, in which the unliganded receptor represses transcription until the time when the $20 \mathrm{E}$ binding relieves repression. The important difference between the inductive and permissive response elements is that, in the absence of receptor - as it is the case in this study - there will be no activation of the gene controlled by the inductive response element, whereas in the case of the permissive element, the repression is absent and the gene is activated precociously.

It was also suggested that EcR and USP monomers separately act as repressors. ${ }^{7,15}$ The observation that EcR $\uparrow$ rescues USP $\downarrow$, suggests that the normal repression by EcR/ USP heterodimers can be efficiently carried out by EcR alone, either acting as a monomer or as a homodimer. Alternatively, in the absence of USP, EcR could heterodimerize with a third, unknown, receptor. That idea was suggested for USP in the eye disc morphogenetic furrow progression because EcR is not required in that system. ${ }^{41}$ However, the suggestion seemed to conflict with data in Manduca ${ }^{42}$ and Drosophila ${ }^{43}$ showing that morphogenetic furrow progression requires ecdysone. The issue was provisionally settled by proposing a novel hormone transduction pathway involving an uncharacterized receptor. The EcR-independent function of USP in the eye would occur via heterodimerization of USP with one of the numerous orphan nuclear receptors identified in Drosophila. ${ }^{44}$ Actually, it has been shown that USP transiently interacts with FoxO in the $P G$, as a nutritional-dependent mechanism to control ecdysone biosynthesis before reaching critical weight (i.e., at $82 \mathrm{~h} \mathrm{AEL).}{ }^{45}$ These proposals would be in line with another study showing that silencing USP in the salivary gland does not block glue protein synthesis but it requires $\mathrm{EcR} .{ }^{46}$ Yet, the binding of ecdysone to a receptor, other than the heterodimer EcR/USP, remained to be proven.

The quantitative data on reaper expression provide an in vivo assay to these proposals. In the PG, but not in the wing disc, reaper becomes de-repressed when EcR or USP are depleted. This transcriptional change is ligand-independent because ecdysone synthesis is abolished in the PG under these conditions, and it can be re-established by EcR alone. Although formally possible, the involvement of a third, unknown, co-repressor would require its strict tissue specificity. The Drosophila homolog of Alien, COUP-TF1, is a corepressor reported to bind EcR but not USP. ${ }^{4,48}$ Out of the current transcriptional cofactors known to bind EcR and USP, histone methyltransferases TRR, ${ }^{49} \mathrm{dSet} 2,{ }^{50} \mathrm{dG} 9 \mathrm{a},{ }^{51}$ nucleosome remodeling factor NURF ${ }^{52}$ and Taiman, ${ }^{53}$ all of them act as co-activators, rather than co-repressors. In any event, assuming co-repressors, in the light of the current data, the search for them should be conducted in the PG. Noticeably, the magnitude of reaper de-repression is different in EcR $\downarrow$ versus USP $\downarrow$, and in prothoracic versus salivary glands. Thus, either the repressing activity of EcR is stronger than that of USP or, alternatively, additional, still unknown, components of the gene repression complex will be required to account for this quantitative feature on reaper expression control.

Anti-estrogen treatments are often considered in several types of human cancers. However, development of resistance, ${ }^{36,37}$ sometimes due to mutations in their receptors, ${ }^{54}$ or undesirable secondary effects ${ }^{55}$ including bone mass loss $^{56}$ represent serious handicaps. The EcR requirement for cell survival shown here in flies is operative in tumor overgrowths, at least those elicited by deregulated $y k i$ or Ras signaling which are conserved in humans. ${ }^{57}$ Notably, the $y k^{*}$ condition, although leading to aberrant overgrowth, does not imply a loss of cell identity as tested for the EcR requirement or, at least, does not imply the de novo acquisition of a requirement for EcR. Taken together, the data reported here suggest that targeting steroid receptors may be an efficient strategy against tumor overgrowth providing that the affected cell type requires that particular receptor for survival. This is in contrast to current strategies that focus on modulating systemic steroid levels. A prior characterization of receptor requirement in the affected tissue may provide more consistent results.

\section{Materials and Methods}

Fly strains. The following fly stocks were obtained from the Bloomington Drosophila Stock Center (Fly Base http://flybase.bio.indiana.edu) except where indicated: usp ${ }^{5}$ (\#44383), UAS-bsk (\#9310), UAS-Atg8-mCherry (\#37750), Df(3 L) H99, knir-1 $p^{p} /$ TM3,Sb (\#1576, a deficiency for reaper and hid), UAS-Ras ${ }^{V 12}$ (\#4847), UAS-p35 (\#5072), UAS-dIAP1(\#6657), tub-Gal80 (\#7108), UAS-EcRARNAi (\#9328), UAS-ECRB1-RNAi (\#9329), UAS-ECRB1 W650A (\#6872), UAS-

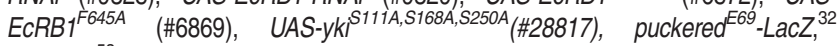
ppl-Gal4, phm-Gal4UAS-CD8-GFP (gift from Mike O'Connor, University of Minnesota), UAS-Torso (gift from Jordi Casanova, Instituto de Recerca Biomédica), UAS- $\beta$-FTZ-f1 (gift from Rosa Barrio, CIC-Biogune, Center for Cooperative Research in Biosciences), UAS-Broad Z1-Z4 isoforms (gift from Lynn Riddiford, Howard Hughes Medical Institute), UAS-Atg4 ${ }^{\text {C98A }}$ (gift from Dolores Ganfornina, University of Valladolid). RNAi lines used were obtained from Vienna Drosophila RNAi Center (VDRC) (https://stockcenter.vdrc.at): UAS-usp-RNAi (\#16893GD), UAS-rpr-RNAi (\#12045GD), UAS-Hid-RNAi (\#7912GD), UAS-Sickle-RNAi (\#102512KK), UAS-Atg1-RNAi (\#16133GD), UAS-Atg5-RNAi (\#104461KK), UAS-Atg7-RNAi (\#45558GD), UAS-EcR-RNAi (\#37058GD), UAS-ptth-RNAi (\#102043KK) and UAS-ECR-RNAi (\#9327). The genotype elav-Gal80; rotundGal4 UAS-GFP ${ }^{n / s}$ was used to drive expression to the salivary gland and imaginal discs without interference from the nervous system expression of the driver

Inmunohistochemistry. Larvae were fixed with $4 \%$ paraformaldehyde in PBS for 20 min, and then washed with PBS. GFP was directly visualized. Anti-Broad core and anti-EcR antibodies were from the Developmental Studies Hybridoma Bank (University of lowa) while anti-caspase-3 was from Cell Signaling (Danvers, MA, USA) and anti-phosphohistone $\mathrm{H} 3$ (Ser10) was from Millipore (Billerica, MA, USA). Primary antibodies were incubated overnight in PBS containing: $0.1 \%$ Triton-X100 5\% normal goat serum and 5\% bovine serum albumin. After washing with PBS, they were incubated with Alexa 488-, 568- or 594-conjugated secondary antibodies (Invitrogen, Carlsbad, CA, USA). For mounting, Vectashield (Vector Laboratories, Burlingame, CA, USA) containing DAPI was used. Images were acquired using a Leica TSC SP5 confocal microscope (Leica, Wetzlar, Germany).

Quantitative RT-PCR. For quantitative RT-PCR assays from whole larvae, RNA from at least five larvae per genotype was extracted using Trizol (Invitrogen). In case of organ-specific qRT-PCR, 20 Ring glands or 10 salivary glands or 20 imaginal wing discs were dissected from each genotype and RNA was extracted using RNeasy micro Kit (QIAGEN, Hilden, Germany). One to $5 \mu \mathrm{g}$ RNA were used for reverse transcription, RT, performed with Supercript II (Invitrogen) according to the manufacturer's instructions. Quantitative PCR was carried out using Taqman MGB probes (Applied Biosystems, Waltham, MA, USA) for the different genes analyzed. RNA polymerase II (RNApollI) was used as a housekeeping gene control. Data were captured on a 7500 Real Time PCR System (Applied Biosystems) and analyzed using relative expression to RNApoll and plotted as a percentage of the respective control larva.

Oil Red 0 staining. Ring glands were fixed in $4 \%$ paraformaldehyde for 20 min, washed twice in PBS and incubated in an Oil Red $O$ (Sigma-Aldrich, 
St Louis, MO, USA) solution at $0.06 \%$ for 30 min. Samples were washed twice with PBS before mounting in Vectashield (Vector Laboratories).

Larval staging and $20 \mathrm{E}$ feeding. The corresponding crosses were allowed to lay eggs in $2 \mathrm{~h}$ batches over agar plates with yeast paste, and this was considered as point $0 \mathrm{~h}$ after egg laying (AEL). Twenty four hours later, all hatched larvae were collected in a new plate and kept at $25^{\circ} \mathrm{C}$ up to the indicated time for harvesting. For 20E feeding: UAS-EC $R^{R N A i}$ or UAS-USP ${ }^{R N A i}$ flies were crossed with phm-Gal4 to knockdown expression in the PG. Experimental and control animals (phm-Gal4 > UAS-LacZ) were collected at $116 \mathrm{~h} \mathrm{AEL}$ and placed in groups of 10 individuals in plates supplemented with 20E (Sigma-Aldrich) dissolved in ethanol at $1 \mathrm{mg} / \mathrm{ml}$ and mixed with yeast. Control larvae were fed with yeast mixed with ethanol.

Ecdysteroid titers. Fifteen to 20 staged larvae 3 were weighed and preserved in $1 \mathrm{ml}$ of methanol. Prior to the assay, samples were homogenized and centrifuged $(10 \mathrm{~min}$ at $18000 \times g)$ twice and the resulting methanol supernatants were dried. Samples were resuspended in $50 \mu$ l of enzyme immunoassay buffer $(0.4 \mathrm{M} \mathrm{NaCl}$, $1 \mathrm{mM}$ EDTA, $0.1 \%$ bovine serum albumin in $0.1 \mathrm{M}$ phosphate buffer). Ecdysteroid levels were quantified by ELISA as described ${ }^{59}$ 20E (Sigma-Aldrich) and 20E-acetylcholinesterase (Cayman Chemical, Ann Arbor, MI, USA) were used as the standard and enzymatic tracer, respectively. The antiserum (Cayman Chemical) was used at a dilution of 1:50 000. Absorbance was read at $450 \mathrm{~nm}$ using a MultiscanPlus II Spectrophotometer (Labsystems, Ramat-Gan, Israel). The ecdysteroid antiserum has the same affinity for ecdysone and $20 \mathrm{E},{ }^{59}$ but because the standard curve was obtained with the latter, results are expressed as $20 \mathrm{E}$ equivalents.

Statistics. All numerical data are presented as average + S.E.M. Statistical significance was calculated using Student's two-tailed $t$-test (unpaired two samples for means) after application of the Kolmogorov-Smirnov method to verify the normality of data distribution. Significance was denoted as ${ }^{* *} P<0.001,{ }^{* *} P<0.01$ and ${ }^{*} P<0.05$.

\section{Conflict of Interest}

The authors declare no conflict of interest.

Acknowledgements. Critical comments from Ferrús' lab members are most appreciated. Research was funded by grants from the Spanish Ministry of Economy BFU2009-12410 and BFU2012-38191 to AF, and BFU2009-10571 to DM.

1. Chang KH, Sharifi N. Prostate cancer-from steroid transformations to clinical translation. Nat Rev Urol 2012; 9: 721-724.

2. Vest RS, Pike CJ. Gender, sex steroid hormones, and Alzheimer's disease. Horm Behav 2012; 63: 301-307.

3. Gilbert LI. Halloween genes encode P450 enzymes that mediate steroid hormone biosynthesis in Drosophila melanogaster. Mol Cell Endocrinol 2004; 215: 1-10.

4. Ou Q, Magico A, King-Jones K. Nuclear receptor DHR4 controls the timing of steroid hormone pulses during Drosophila development. PLoS Biol 2011; 9: e1001160.

5. Yao TP, Forman BM, Jiang Z, Cherbas L, Chen JD, McKeown M et al. Functional ecdysone receptor is the product of EcR and Ultraspiracle genes. Nature 1993; 366: 476-479.

6. Talbot WS, Swyryd EA, Hogness DS. Drosophila tissues with different metamorphic responses to ecdysone express different ecdysone receptor isoforms. Cell 1993; 73: 1323-1337.

7. Cherbas L, Hu X, Zhimulev I, Belyaeva E, Cherbas P. EcR isoforms in Drosophila: testing tissue-specific requirements by targeted blockade and rescue. Development 2003; 130: 271-284.

8. Davis MB, Carney GE, Robertson AE, Bender M. Phenotypic analysis of EcR-A mutants suggests that $\mathrm{EcR}$ isoforms have unique functions during Drosophila development. Dev Biol 2005; 282: 385-96.

9. Sung $C$, Robinow $S$. Characterization of the regulatory elements controlling neuronal expression of the A-isoform of the ecdysone receptor gene of Drosophila melanogaster. Mech Dev 2000; 91: 237-248.

10. Gradilla AC, Mansilla A, Ferrus A. Isoform-specific regulation of a steroid hormone nuclear receptor by an E3 ubiquitin ligase in Drosophila melanogaster. Genetics 2011; 189: 871-883.

11. Vogtli M, Elke C, Imhof MO, Lezzi M. High level transactivation by the ecdysone receptor complex at the core recognition motif. Nucleic Acids Res 1998; 26: 2407-2414.
12. Weston $A D$, Blumberg $B$, Underhill TM. Active repression by unliganded retinoid receptors in development: less is sometimes more. J Cell Biol 2003; 161: 223-228.

13. Ghbeish N, McKeown M. Analyzing the repressive function of ultraspiracle, the Drosophila RXR, in Drosophila eye development. Mech Dev 2002; 111: 89-98.

14. Schubiger M, Carre C, Antoniewski C, Truman JW. Ligand-dependent de-repression via $\mathrm{EcR} / \mathrm{USP}$ acts as a gate to coordinate the differentiation of sensory neurons in the Drosophila wing. Development 2005; 132: 5239-5248

15. Schubiger M, Truman JW. The RXR ortholog USP suppresses early metamorphic processes in Drosophila in the absence of ecdysteroids. Development 2000; 127: 1151-1159.

16. Huet F, Ruiz C, Richards G. Sequential gene activation by ecdysone in Drosophila melanogaster: the hierarchical equivalence of early and early late genes. Development 1995; 121: 1195-1204.

17. Ashburner M. Sequential gene activation by ecdysone in polytene chromosomes of Drosophila melanogaster. I. Dependence upon ecdysone concentration. Dev Biol 1973; 35 47-61.

18. Gompel A, Somai S, Chaouat M, Kazem A, Kloosterboer HJ, Beusman I et al. Hormonal regulation of apoptosis in breast cells and tissues. Steroids 2000; 65: 593-598.

19. McDaniel RE, Maximov PY, Jordan VC. Estrogen-mediated mechanisms to control the growth and apoptosis of breast cancer cells: a translational research success story. Vitam Horm 2013; 93: 1-49.

20. Cordenonsi M, Zanconato F, Azzolin L, Forcato M, Rosato A, Frasson C et al. The Hippo transducer TAZ confers cancer stem cell-related traits on breast cancer cells. Cell 2011; 147: 759-772.

21. Zhang X, George J, Deb S, Degoutin JL, Takano EA, Fox SB et al. The Hippo pathway transcriptional co-activator, YAP, is an ovarian cancer oncogene. Oncogene 2011; 30 2810-2822.

22. Staley BK, Irvine KD. Hippo signaling in Drosophila: recent advances and insights. Dev Dyn 2012; 241: 3-15.

23. Miller DL, Ballard SL, Ganetzky B. Analysis of synaptic growth and function in Drosophila with an extended larval stage. J Neurosci 2012; 32: 13776-13786.

24. Karim FD, Guild GM, Thummel CS. The Drosophila Broad-Complex plays a key role in controlling ecdysone-regulated gene expression at the onset of metamorphosis. Development 1993; 118: 977-988.

25. Wendler F, Gillingham AK, Sinka R, Rosa-Ferreira C, Gordon DE, Franch-Marro X et al A genome-wide RNA interference screen identifies two novel components of the metazoan secretory pathway. EMBO J 2010; 29: 304-314.

26. Dai JD, Gilbert LI. Metamorphosis of the corpus allatum and degeneration of the prothoracic glands during the larval-pupal-adult transformation of Drosophila melanogaster: a cytophysiological analysis of the ring gland. Dev Biol 1991; 144: 309-326.

27. Komatsu M, Waguri S, Ueno T, Iwata J, Murata S, Tanida I et al. Impairment of starvationinduced and constitutive autophagy in Atg7-deficient mice. J Cell Biol 2005; 169: 425-434.

28. Rewitz KF, Yamanaka N, Gilbert LI, O'Connor MB. The insect neuropeptide PTTH activates receptor tyrosine kinase torso to initiate metamorphosis. Science 2009; 326: 1403-1405.

29. Lee CY, Wendel DP, Reid P, Lam G, Thummel CS, Baehrecke EH. E93 directs steroidtriggered programmed cell death in Drosophila. Mol Cell 2000; 6: 433-443.

30. Baehrecke EH. Steroid regulation of programmed cell death during Drosophila development. Cell Death Differ 2000; 7: 1057-1062.

31. Maiuri MC, Zalckvar E, Kimchi A, Kroemer G. Self-eating and self-killing: crosstalk between autophagy and apoptosis. Nat Rev Mol Cell Biol 2007; 8: 741-752.

32. Adachi-Yamada T, Fujimura-Kamada K, Nishida Y, Matsumoto K. Distortion of proximodistal information causes JNK-dependent apoptosis in Drosophila wing. Nature 1999; 400: 166-169.

33. Kanda H, Miura M. Regulatory roles of JNK in programmed cell death. J Biochem 2004; 136 : $1-6$

34. Boutros M, Paricio N, Strutt DI, Mlodzik M. Dishevelled activates JNK and discriminates between JNK pathways in planar polarity and wingless signaling. Cell 1998; 94 109-118.

35. Jiang C, Lamblin AF, Steller H, Thummel CS. A steroid-triggered transcriptional hierarchy controls salivary gland cell death during Drosophila metamorphosis. Mol Cell 2000; 5 : 445-455.

36. Musgrove EA, Sutherland RL. Biological determinants of endocrine resistance in breast cancer. Nat Rev Cancer 2009; 9: 631-643.

37. Suzuki $\mathrm{H}$, Ueda $\mathrm{T}$, Ichikawa $\mathrm{T}$, Ito $\mathrm{H}$. Androgen receptor involvement in the progression of prostate cancer. Endocr Relat Cancer 2003; 10: 209-216.

38. Zhang W, Cohen SM. The Hippo pathway acts via p53 and microRNAs to control proliferation and proapoptotic gene expression during tissue growth. Biol Open 2013; 2 $822-828$

39. Kang Y, Bashirullah A. A steroid-controlled global switch in sensitivity to apoptosis during Drosophila development. Dev Biol 2014; 386: 34-41.

40. Niwa R, Matsuda T, Yoshiyama T, Namiki T, Mita K, Fujimoto $Y$ et al. CYP306A1, a cytochrome P450 enzyme, is essential for ecdysteroid biosynthesis in the prothoracic glands of Bombyx and Drosophila. J Biol Chem 2004; 279: 35942-35949.

41. Brennan CA, Li TR, Bender M, Hsiung F, Moses K. Broad-complex but not ecdysone receptor, is required for progression of the morphogenetic furrow in the Drosophila eye. Development 2001; 128: 1-11.

42. Champlin DT, Truman JW. Ecdysteroids govern two phases of eye development during metamorphosis of the moth, Manduca sexta. Development 1998; 125: 2009-2018. 
43. Brennan CA, Ashburner M, Moses K. Ecdysone pathway is required for furrow progression in the developing Drosophila eye. Development 1998; 125: 2653-2664.

44. Sullivan AA, Thummel CS. Temporal profiles of nuclear receptor gene expression reveal coordinate transcriptional responses during Drosophila development. Mol Endocrinol 2003; 17: 2125-2137.

45. Koyama T, Rodrigues MA, Athanasiadis A, Shingleton AW, Mirth CK. Nutritional control of body size through FoxO-Ultraspiracle mediated ecdysone biosynthesis. Elife 2014; 3.

46. Costantino BF, Bricker DK, Alexandre K, Shen K, Merriam JR, Antoniewski C et al. A novel ecdysone receptor mediates steroid-regulated developmental events during the mid-third instar of Drosophila. PLOS Genet 2008; 4: e1000102.

47. Dressel U, Thormeyer D, Altincicek B, Paululat A, Eggert M, Schneider S et al. Alien, a highly conserved protein with characteristics of a corepressor for members of the nuclear hormone receptor superfamily. Mol Cell Biol 1999; 19: 3383-3394.

48. Papaioannou M, Melle C, Baniahmad A. The coregulator Alien. Nucl Recept Signal 2007; 5: e008.

49. Sedkov Y, Cho E, Petruk S, Cherbas L, Smith ST, Jones RS et al. Methylation at lysine 4 of histone H3 in ecdysone-dependent development of Drosophila. Nature 2003; 426: 78-83.

50. Stabell M, Larsson J, Aalen RB, Lambertsson A. Drosophila dSet2 functions in H3-K36 methylation and is required for development. Biochem Biophys Res Commun 2007; 359: 784-789.

51. Stabell M, Bjorkmo M, Aalen RB, Lambertsson A. The Drosophila SET domain encoding gene dEset is essential for proper development. Hereditas 2006; 143: 177-188.
52. Badenhorst $\mathrm{P}$, Xiao H, Cherbas L, Kwon SY, Voas M, Rebay I et al. The Drosophila nucleosome remodeling factor NURF is required for Ecdysteroid signaling and metamorphosis. Genes Dev 2005; 19: 2540-2545.

53. Bai J, Uehara Y, Montell DJ. Regulation of invasive cell behavior by taiman, a Drosophila protein related to AIB1, a steroid receptor coactivator amplified in breast cancer. Cell 2000; 103: $1047-1058$.

54. Clarke R, Liu MC, Bouker KB, Gu Z, Lee RY, Zhu Y et al. Antiestrogen resistance in breast cancer and the role of estrogen receptor signaling. Oncogene 2003; 22: 7316-7339.

55. Grossmann M, Cheung AS, Zajac JD. Androgens and prostate cancer; pathogenesis and deprivation therapy. Best Pract Res Clin Endocrinol Metab 2013; 27: 603-616.

56. Saad F, Adachi JD, Brown JP, Canning LA, Gelmon KA, Josse RG et al. Cancer treatment-induced bone loss in breast and prostate cancer. J Clin Oncol 2008; 26: 5465-5476.

57. Matallanas D, Romano D, Hamilton G, Kolch W, O'Neill E. A Hippo in the ointment: MST signalling beyond the fly. Cell Cycle 2008; 7: 879-884.

58. Colombani J, Raisin S, Pantalacci S, Radimerski T, Montagne J, Leopold P. A nutrient sensor mechanism controls Drosophila growth. Cell 2003; 114: 739-749.

59. Pankotai T, Popescu C, Martin D, Grau B, Zsindely N, Bodai L et al. Genes of the ecdysone biosynthesis pathway are regulated by the dATAC histone acetyltransferase complex in Drosophila. Mol Cell Biol 2010; 30: 4254-4266.

\section{Supplementary Information accompanies this paper on Cell Death and Differentiation website (http://www.nature.com/cdd)}

